\title{
q-Iterative Methods
}

\author{
Prashant Singh ${ }^{1}$, Pramod Kumar Mishra ${ }^{2}$, R.S.Pathak ${ }^{3}$ \\ ${ }^{I}$ (Department of Computer Science, Banaras Hindu University, India) \\ ${ }^{2}$ (Deparment of Computer Science, Banaras Hindu University, India) \\ ${ }^{3}$ (D.S.T. Centre for Interdisciplinary Mathematical Sciences ,Banaras Hindu University,India)
}

Abstract: This paper is devoted to derivation of q-analogues of Iterative Methods for solution of algebraic and transcendental equations and comparing accuracy of results with classical methods.

Keywords: Basic, q-special function, q-analogue

\section{Introduction}

A q-hypergeometric function is an analogue of an ordinary hypergeometric function with addition of an extra parameter $\mathrm{q}$, where $0<\mathrm{q}<1$. When $\mathrm{q}$ tends to one, the $\mathrm{q}$-hyper geometric function tends to normal hypergeometric function. Results on basic differentiations, integrations, basic transformations and identities, basic analogue of certain classical functions and their applications are available in the literature .q-analogue finds applications in a number of areas, including the study of fractals and multi-fractal measures, and expressions for the entropy of chaotic dynamical systems. The word q-analogue and basic analogue are synonyms.

\subsection{Basic Number System}

$$
[\alpha ; q]=\frac{1-q^{\alpha}}{1-q}=1+\mathrm{q}+q^{2}+\cdots+\mathrm{q}^{(\alpha-1)}
$$

\subsection{Basic Factorial Notation}

$[\mathrm{n} ; \mathrm{q}] !=[\mathrm{n} ; \mathrm{q}][\mathrm{n}-1 ; \mathrm{q}] \ldots[3 ; \mathrm{q}][2 ; \mathrm{q}][1 ; \mathrm{q}]$

\section{3 q-exponential function}

$$
\begin{aligned}
& E_{q}(x)=\sum_{r=0}^{\infty} x^{r} /[r ; q] ! \\
& E_{q}^{-1}(x)=\sum_{r=0}^{\infty} x^{r} q^{\frac{r(r-1)}{2}} /[r ; q] ! \\
& E(q, x)=\sum_{r=0}^{\infty} x^{r} q^{\frac{r(r-1)}{4}} /[r ; q] !
\end{aligned}
$$

\subsection{Basic Differentiation operator}

$$
\mathrm{D}_{\mathrm{q}, \mathrm{x}} \mathrm{f}(\mathrm{x})=\frac{\mathrm{f}(\mathrm{qx})-\mathrm{f}(\mathrm{x})}{x(q-1)}
$$

Formula for $\mathrm{q}$ differentiation by parts

$$
\begin{aligned}
& D_{\mathrm{q}, \mathrm{x}}(\mathrm{f}(\mathrm{x}) \mathrm{g}(\mathrm{x}))=\mathrm{g}(\mathrm{x}) \mathrm{D}_{\mathrm{q}, \mathrm{x}} \mathrm{f}(\mathrm{x})+\mathrm{f}(\mathrm{x}) \mathrm{D}_{\mathrm{q}, \mathrm{x}} \mathrm{g}(\mathrm{x}) \\
& \mathrm{D}_{\mathrm{q}, \mathrm{x}} \frac{f(x)}{g(x)}=\frac{\mathrm{g}(\mathrm{x}) \mathrm{D}_{\mathrm{q}, \mathrm{x}} \mathrm{f}(\mathrm{x})-\mathrm{f}(\mathrm{x}) \mathrm{D}_{\mathrm{q}, \mathrm{x}} \mathrm{g}(\mathrm{x})}{\mathrm{g}(\mathrm{x}) \mathrm{g}(\mathrm{q} x)}
\end{aligned}
$$

1.5 Ward-Alsalam [5] q differentiation operator containing two parameters

$$
\begin{aligned}
& D_{q_{1} q_{2}} \mathrm{f}(\mathrm{x})=\frac{f\left(q_{1} x\right)-f\left(q_{2} x\right)}{x\left(q_{1}-q_{2}\right)} \text { where } q_{1}=\mathrm{q}_{2}^{-1} \\
& D D_{q_{1} q_{2}} f(x)=\frac{\left[q_{2} f\left(q_{1}^{2} x\right)+q_{1} f\left(q_{2}^{2} x\right)-q_{1} \mathrm{f}\left(q_{1} q_{2} x\right)-q_{2} \mathrm{f}\left(q_{1} q_{2} x\right)\right]}{\left(q_{1}-q_{2}\right)^{2} x^{2}}
\end{aligned}
$$

1.6 Basic analogue of Taylor's Theorem

Jackson(1909) introduced q analogue of Taylor's Theorem

$f(x)=f(a)+\frac{(x-a)^{(1)}}{[1: q]} D_{q} f(a)+\frac{(x-a)^{(2)}}{[2 ; q] !} D_{q}^{2} f(a)+\ldots+\frac{(x-a)^{(n)}}{[n ; q] !} D_{q}^{n} \mathrm{f}(\mathrm{a})$, where

$\mathrm{R}_{\mathrm{n}}=\frac{(x-a)^{(n+1)}}{[n+1 ; q] !} D^{(n+1)} \mathrm{f}(\xi)$, where $\xi$ lies between $x$ and $a$. 
It can be rewritten as

$f(x)=\sum_{n=0}^{\infty} \frac{(1-q)^{n}}{(q ; q)_{n}} D_{q}^{n} f(a)[x-a]_{n}$

For double parameter it can be written as

$f(x)=f(a)+\frac{(x-a)^{(1)}}{[1: q] !} D_{q_{1} q_{2}} f(a)+\frac{(x-a)^{(2)}}{[2 ; q] !} D_{q_{1} q_{2}}^{2} f(a)+\ldots+\frac{(x-a)^{(n)}}{[n ; q] !} D_{q_{1} q_{2}}^{n} \mathrm{f}(\mathrm{a})$

where $\mathrm{q}_{1}$ and $\mathrm{q}_{2}$ are inverses of each other.

2.1 q-analogue of Newton Raphson Method

q-analogue of Iterative Methods

$x_{n+1}=x_{n}-\frac{f\left(x_{n}\right) x_{n}(1-q)}{f\left(x_{n}\right)-f\left(q x_{n}\right)}$

2.2 q-analogue of Newton Raphson Method having multiplicity

$x_{n+1}=x_{n}-m \frac{f\left(x_{n}\right) x_{n}(1-q)}{f\left(x_{n}\right)-f\left(q x_{n}\right)}$, where $m$ is multiplicity.

2.3 q-analogue of Newton Raphson Method for multiple root

$\mathrm{x}_{\mathrm{k}+1}=\mathrm{x}_{\mathrm{k}}-\frac{m(3-m)^{1}}{[2 ; q]} \frac{f(k)}{D_{q} f(k)}-\frac{m^{2}}{[2 ; q]}\left(\frac{f(k)}{D_{q} f(k)}\right)^{2} \frac{D_{q}^{2} f(k)}{D_{q} f(k)}$

2.4 q-analogue of Newton Raphson Method for complex root

For complex root Newton Raphson method will look like

$z_{n+1}=z_{n}-\frac{f\left(z_{n}\right) z_{n}(1-q)}{f\left(z_{n}\right)-f\left(q z_{n}\right)}$

and initial approximation $\mathrm{z}_{\mathrm{o}}$ must be complex.

After finding one root $\mathrm{z}_{1}$, it can be applied on the deflated polynomial

$f^{*}(z)=\frac{f(z)}{z-z_{1}}$,If number of roots are k, new iteration should be applied on

$f^{*}(\mathrm{z})=\frac{f(z)}{\left(z-z_{1}\right)\left(z-z_{2}\right) \ldots\left(z-z_{k}\right)}$

So, $z_{n+1}=z_{n}-\frac{f^{*}\left(z_{n}\right) z_{n}(1-q)}{f^{*}\left(z_{n}\right)-f^{*}\left(q z_{n}\right)}$

2.5 q-analogue of Chebyshev Method

$\mathrm{f}(\mathrm{x})=\mathrm{f}\left(\mathrm{x}_{\mathrm{k}}+\mathrm{x}-\mathrm{x}_{\mathrm{k}}\right)$ and approximating $\mathrm{f}(\mathrm{x})$ by second degree Taylor's Series Expansion about point $\mathrm{x}_{\mathrm{k}}$ we get,

$\mathrm{x}_{\mathrm{k}+1}=\mathrm{x}_{\mathrm{k}}-\frac{f(k)}{D_{q} f(k)}-\frac{1}{[2 ; q]}\left(\mathrm{x}_{\mathrm{k}+1}-\mathrm{x}_{\mathrm{k}}\right)^{2} \frac{D_{q}^{2} f(k)}{D_{q} f(k)}$

Also, we can write it like

$\mathrm{x}_{\mathrm{k}+1}=\mathrm{x}_{\mathrm{k}}-\frac{f(k)}{D_{q} f(k)}-\frac{1}{[2 ; q]}\left(\frac{f(k)}{D_{q} f(k)}\right) \frac{2_{q}^{2} f(k)}{D_{q} f(k)}$

By simplifying we can write this equation as

$x_{n+1}=x_{n-} \frac{x_{n} f\left(x_{n}\right)(1-q)}{f\left(x_{n}\right)-f\left(q x_{n}\right)}-\frac{(1-q) x_{n} f\left(x_{n}\right)\left[f\left(q^{2} x_{n}\right)-[2 ; q] f\left(q x_{n}\right)+q f\left(x_{n}\right)\right.}{[2 ; q] q\left(f\left(x_{n}\right)-f\left(q x_{n}\right)\right)^{3}}$

2.6 q-analogue of Newton Raphson Method with two parameters

$x_{k+1=} x_{k}-\frac{f\left(x_{k}\right)}{f\left(q_{1} x_{k}\right)-f\left(q_{2} x_{k}\right)}\left(q_{1}-q_{2}\right) \mathrm{x}_{\mathrm{k}}$

2.7 q-analogue of Newton Raphson Method for multiple roots

$x_{k+1=} x_{k}-\frac{f\left(x_{k}\right)}{f\left(q_{1} x_{k}\right)-f\left(q_{2} x_{k}\right)} \mathrm{m}\left(q_{1}-q_{2}\right) \mathrm{x}_{\mathrm{k}}$

2.8 q-analogue of Chebyshev Method having two parameters

$\mathrm{X}_{\mathrm{k}+1}=\mathrm{x}_{\mathrm{k}}-\frac{f(k)}{D_{q} f(k)}-\frac{1}{[2 ; q]}\left(\frac{f(k)}{D_{q} f(k)}\right)^{2} \frac{D_{q}^{2} f(k)}{D_{q} f(k)}$

$x_{k+1}=x_{k-} \frac{f\left(x_{k}\right)\left(q_{1}-q_{2}\right) f\left(x_{k}\right)}{f\left(q_{1} x_{k}\right)-f\left(q_{2} x_{k}\right)}-\frac{1}{[2 ; q]}\left(\frac{f\left(x_{k}\right)\left(q_{1}-q_{2}\right) f\left(x_{k}\right)(q 1-q 2)}{f\left(q_{1} x_{k}\right)-f\left(q_{2} x_{k}\right)}\right)^{2}\left(\frac{q_{2} f\left(q_{1}^{2} x\right)+q_{1} f\left(q_{2}^{2} x\right)-q_{1} \mathrm{f}\left(q_{1} q_{2} x\right)-q_{2} \mathrm{f}\left(q_{1} q_{2} x\right)}{\left(q_{1}-q_{2}\right) x_{k}} \frac{\mathrm{xk}}{f\left(q_{1} x_{k}\right)-f\left(q_{2} x_{k}\right)}\right)$ 
2.9 q-analogue Multipoint Iteration Method with two parameters

$x_{k+1}^{*}=x_{k}-\frac{f\left(x_{k}\right)}{f\left(q_{1} x_{k}\right)-f\left(q_{2} x_{k}\right)} \frac{\left(q_{1}-q_{2}\right) \mathrm{x}_{\mathrm{k}}}{[2 ; q]}$

$x_{k+1}=x_{k}-\frac{f\left(x_{k}\right)}{f\left(q_{1} x_{k+1}^{*}\right)-f\left(x_{k+1}^{*} q_{2}\right)} \frac{\left(q_{1}-q_{2}\right) x_{k+1}^{*}}{[2 ; q]}$

Second Method

$x_{k+1}^{*}=x_{k}-\frac{f\left(x_{k}\right)}{f\left(q_{1} x_{k}\right)-f\left(q_{2} x_{k}\right)} \frac{\left(q_{1}-q_{2}\right) x_{k}}{[1 ; q]}$
$x_{k+1}=x_{k}-\frac{f\left(x_{k+1}^{*}\right)}{f\left(q_{1} x_{k}\right)-f\left(x_{k} q_{2}\right)} \frac{\left(q_{1}-q_{2}\right) x_{k}}{[1 ; q]}$

PROBLEM 1(solution by q method with double parameter)

Let us take a problem $x e^{x}-1=0$, let us take $\mathrm{x}_{0}=1$

Table 1: Calculation of $\mathrm{x}_{1}$ by iterative method ( $\mathrm{q}$-analogue of Numerical Methods) for different values of $\mathrm{q}_{1}$

\begin{tabular}{|c|c|c|c|c|c|}
\hline Value of $q_{1}$ & Value of $q_{2}$ & $\mathbf{f}\left(\mathbf{q}_{1} \mathbf{x}_{0}\right)$ & $\mathbf{f}\left(\mathbf{q}_{2} \mathbf{x}_{0}\right)$ & $\operatorname{Dq}_{1} \mathbf{q}_{2} \mathbf{f}(\mathbf{x})$ & $\mathbf{x}_{1}$ \\
\hline 0.99999999 & 1.00000001 & 1.718281774 & 1.718281883 & 5.436563675 & 0.683939722 \\
\hline 0.999 & 1.001001001 & 1.71284934 & 1.723727922 & 5.436569552 & 0.683940063 \\
\hline 0.98 & 1.020408163 & 1.611167117 & 1.830945828 & 5.438968093 & 0.684079443 \\
\hline 0.97 & 1.030927835 & 1.558806126 & 1.890377286 & 5.442030897 & 0.684257245 \\
\hline 0.96 & 1.041666667 & 1.507228614 & 1.952016987 & 5.446388237 & 0.684509852 \\
\hline 0.95 & 1.052631579 & 1.456424176 & 2.015980164 & 5.452083986 & 0.684839443 \\
\hline 0.94 & 1.063829787 & 1.406382533 & 2.082389756 & 5.459164858 & 0.685248225 \\
\hline 0.93 & 1.075268817 & $\mathbf{1 . 3 5 7 0 9 3 5 3 5}$ & 2.151377031 & 5.467680616 & 0.685738442 \\
\hline 0.92 & 1.086956522 & 1.308547159 & 2.223082277 & 5.477684304 & 0.686312366 \\
\hline 0.91 & 1.098901099 & 1.260733505 & 2.297655556 & 5.489232495 & 0.686972299 \\
\hline 0.9 & 1.111111111 & 1.2136428 & 2.375257531 & 5.502385566 & 0.68772057 \\
\hline 0.89 & 1.123595506 & 1.16726539 & 2.45606038 & 5.517207992 & 0.688559534 \\
\hline 0.88 & 1.136363636 & 1.121591742 & 2.540248803 & 5.533768677 & 0.689491569 \\
\hline 0.87 & 1.149425287 & 1.076612443 & 2.628021124 & 5.552141311 & 0.690519075 \\
\hline 0.86 & 1.162790698 & 1.032318197 & 2.719590522 & 5.57240476 & 0.691644469 \\
\hline 0.85 & 1.176470588 & 0.988699824 & 2.815186379 & 5.594643501 & 0.692870184 \\
\hline 0.84 & 1.19047619 & 0.94574826 & 2.915055782 & 5.618948091 & 0.694198665 \\
\hline 0.83 & 1.204819277 & 0.903454554 & 3.019465185 & 5.645415698 & 0.695632364 \\
\hline 0.82 & 1.219512195 & 0.861809867 & 3.128702256 & 5.674150669 & 0.69717374 \\
\hline 0.81 & 1.234567901 & 0.820805469 & 3.243077929 & 5.705265171 & 0.698825247 \\
\hline 0.8 & 1.25 & 0.780432743 & 3.362928697 & 5.738879898 & $\mathbf{0 . 7 0 0 5 8 9 3 3 8}$ \\
\hline 0.79 & 1.265822785 & 0.740683177 & 3.488619163 & 5.775124844 & 0.702468453 \\
\hline 0.78 & 1.282051282 & 0.701548367 & 3.620544898 & 5.814140178 & 0.704465015 \\
\hline 0.77 & 1.298701299 & 0.663020015 & 3.759135637 & 5.856077201 & 0.706581425 \\
\hline 0.76 & 1.315789474 & 0.625089928 & 3.904858867 & 5.901099418 & 0.708820051 \\
\hline 0.75 & 1.333333333 & 0.587750012 & 4.05822386 & 5.949383738 & 0.711183224 \\
\hline 0.74 & 1.351351351 & 0.550992281 & 4.219786205 & 6.001121803 & 0.713673229 \\
\hline 0.73 & 1.369863014 & 0.514808844 & 4.390152936 & 6.056521489 & 0.716292292 \\
\hline 0.72 & 1.388888889 & 0.479191912 & 4.569988314 & 6.115808574 & 0.719042575 \\
\hline 0.71 & 1.408450704 & 0.444133794 & 4.760020382 & 6.17922863 & 0.721926161 \\
\hline 0.7 & 1.428571429 & 0.409626895 & 4.961048405 & 6.247049131 & 0.724945043 \\
\hline 0.69 & 1.449275362 & 0.375663718 & 5.173951334 & 6.319561854 & 0.728101114 \\
\hline 0.68 & 1.470588235 & 0.342236858 & 5.399697452 & 6.397085573 & 0.731396148 \\
\hline 0.67 & 1.492537313 & 0.309339005 & $\mathbf{5 . 6 3 9 3 5 5 3 9 7}$ & 6.479969121 & $\mathbf{0 . 7 3 4 8 3 1 7 8 8}$ \\
\hline 0.66 & 1.515151515 & 0.276962941 & 5.894106796 & 6.56859487 & 0.738409529 \\
\hline 0.65 & 1.538461538 & 0.245101539 & 6.165260763 & 6.663382676 & 0.7421307 \\
\hline 0.55 & 1.818181818 & -0.04671084 & 10.20117652 & 8.080771393 & 0.787361658 \\
\hline 0.54 & 1.851851852 & -0.073356294 & 10.79927385 & 8.28800152 & 0.792678389 \\
\hline 0.53 & 1.886792453 & -0.099565876 & 11.44937879 & 8.511946423 & 0.798132913 \\
\hline 0.52 & 1.923076923 & -0.125345622 & 12.15765068 & 8.754328507 & 0.80372203 \\
\hline 0.51 & 1.960784314 & -0.150701491 & 12.93117127 & 9.01710381 & 0.809441938 \\
\hline 0.5 & 2 & -0.175639365 & $\mathbf{1 3 . 7 7 8 1 1 2 2}$ & 9.302501042 & 0.815288187 \\
\hline
\end{tabular}

Table 2: Calculation of $\mathrm{x}_{2}$ by iterative method (q-analogue of Numerical Methods) for different values of $\mathrm{q}_{1}$

\begin{tabular}{|l|l|l|l|l|l|l|}
\hline Value of $q_{1}$ & Value of $q_{2}$ & $f\left(q_{1} \times 1\right)$ & $f\left(q_{2} \times 1\right)$ & $D q_{1} q_{2} f(x 1)$ & $f(x 1)$ & $x 2$ \\
\hline 0.99999999 & 1.00000001 & 1.718281665 & 1.718281774 & 5.436563495 & 1.71828172 & 0.683939711 \\
\hline 0.999 & 1.001001001 & 0.353062621 & 0.357629544 & 3.337016362 & 0.35534369 & 0.577454612 \\
\hline 0.98 & 1.020408163 & 0.310637846 & 0.402928352 & 3.338730616 & 0.35580886 & 0.577509342 \\
\hline
\end{tabular}


q-Iterative Methods

\begin{tabular}{|l|l|l|l|l|l|l|}
\hline 0.97 & 1.030927835 & 0.288977125 & 0.428261121 & 3.340919925 & 0.3564024 & 0.577579321 \\
\hline 0.96 & 1.041666667 & 0.267764639 & 0.454701682 & 3.344035159 & 0.35724595 & 0.577679052 \\
\hline 0.95 & 1.052631579 & 0.246990835 & 0.482316489 & 3.348108305 & 0.35834709 & 0.577809725 \\
\hline 0.94 & 1.063829787 & 0.226646219 & 0.511176888 & 3.353173608 & 0.3597136 & 0.577972657 \\
\hline 0.93 & 1.075268817 & 0.206721351 & 0.541359552 & 3.359267741 & 0.36135352 & 0.578169309 \\
\hline 0.92 & 1.086956522 & 0.187206843 & 0.572946954 & 3.366430002 & 0.36327508 & 0.57840129 \\
\hline 0.91 & 1.098901099 & 0.168093349 & 0.606027895 & 3.374702518 & 0.36548679 & 0.57867037 \\
\hline 0.9 & 1.111111111 & 0.149371562 & 0.640698081 & 3.384130478 & 0.36799737 & 0.578978494 \\
\hline
\end{tabular}

Table 3: Calculation of $\mathrm{x} 3$ by iterative method ( $\mathrm{q}$-analogue of Numerical Methods) for different values of $\mathrm{q}_{1}$

\begin{tabular}{|l|l|l|l|l|}
\hline $\mathrm{f}\left(\mathrm{q}_{1} \mathrm{x}_{2}\right)$ & $\mathrm{f}\left(\mathrm{q}_{2} \mathrm{X}_{2}\right)$ & $D q_{1} \mathrm{q}_{2} \mathbf{f}\left(\mathrm{x}_{2}\right)$ & $\mathrm{f}\left(\mathrm{x}_{2}\right)$ & $\mathrm{x}_{3}$ \\
\hline 0.355342497 & 0.355342542 & 3.337012114 & 0.35534252 & 0.577454476 \\
\hline 0.027112248 & 0.030359438 & 2.810233997 & 0.028734265 & 0.567229746 \\
\hline-0.00326886 & 0.062332853 & 2.81116954 & 0.028888077 & 0.567233165 \\
\hline-0.01893519 & 0.080033774 & 2.812364816 & 0.029084763 & 0.567237575 \\
\hline-0.03438198 & 0.098377586 & 2.81406652 & 0.02936511 & 0.567243936 \\
\hline-0.04961177 & 0.117398702 & 2.8162931 & 0.029732504 & 0.567252406 \\
\hline-0.06462693 & 0.137134175 & 2.819064585 & 0.03019071 & 0.567263179 \\
\hline-0.07942957 & 0.157623957 & 2.82240272 & 0.030743904 & 0.567276497 \\
\hline-0.09402159 & 0.178911181 & 2.826331118 & 0.031396709 & 0.567292645 \\
\hline-0.10840467 & 0.201042479 & 2.830875412 & 0.032154223 & 0.567311967 \\
\hline-0.12258028 & 0.224068332 & 2.836063442 & 0.033022065 & 0.567334867 \\
\hline
\end{tabular}

Proceeding in this way we get $x_{4}=0.5671433$ which is required solution.

PROBLEM 2( solution by q method with single parameter)

Basic Analogue of Newton Raphson Method:

Let us solve same problem $f(x)=x e^{x}-1$

Let $\mathrm{x}_{0}=1$

$\mathrm{f}(1)=(\mathrm{e}-1)$

$f(q)=q e^{q}-1$

Table 4: Calculation of $\mathrm{x} 1$ by iterative method (q-analogue of Numerical Methods) for different values of $\mathrm{q}$

\begin{tabular}{|l|l|}
\hline$q$ & $\mathrm{x}_{1}$ \\
\hline 0.96 & 0.6743415 \\
\hline 0.97 & 0.676762956 \\
\hline 0.98 & 0.679169779 \\
\hline 0.99 & 0.681562017 \\
\hline 0.999999999999 & 0.683945343 \\
\hline 1.01 & 0.686302938 \\
\hline 1.09 & 0.704693177 \\
\hline 0.95 & 0.671905363 \\
\hline 0.9 & 0.659502787 \\
\hline 0.8 & 0.633569653 \\
\hline 0.7 & 0.606095896 \\
\hline 0.6 & 0.577041065 \\
\hline 0.5 & 0.546369238 \\
\hline 0.4 & 0.514049563 \\
\hline 0.3 & 0.480056757 \\
\hline 0.2 & 0.444371563 \\
\hline 0.1 & 0.40698115 \\
\hline
\end{tabular}

Value of $\mathrm{x}_{1}$ by Newton Raphson Method is 0.6839397.

$\mathrm{f}\left(\mathrm{x}_{1}\right)$ by Newton Raphson Method is 0.3553424

$\mathrm{f}\left(\mathrm{x}_{1}\right)$ by $\mathrm{q}$ analogue ot Newton Raphson Method at $\mathrm{q}=0.99$ is 0.347423143

$\mathrm{f}\left(\mathrm{x}_{1}\right)$ by $\mathrm{q}$ method at $\mathrm{q}=0.97$ is 0.33153 


\section{Observation}

We can observe that for $q$ tending to one we get more accurate value of $x_{1}$ for which $f\left(x_{1}\right)$ is closer to zero in comparison with classical method. $\mathrm{f}\left(\mathrm{x}_{1}\right)$ at $\mathrm{q}=0.99$ is 0.347423143 and $\mathrm{f}\left(\mathrm{x}_{1}\right)$ by Newton Raphson method is 0.3553424 . It is apparent that $\mathrm{f}\left(\mathrm{x}_{1)}\right.$ by q-analogue of Newton Raphson method is closer to zero in comparison with $\mathrm{f}\left(\mathrm{x}_{1}\right)$ calculated by Newton Raphson Method method

\section{PROBLEM 3(Example of q-analogue of generalised Newton Raphson Method and q analogue of Newton Raphson Method)}

Let us take $f(x)=x^{3}-x^{2}-x+1=0$

$\mathrm{x}_{0}=0.8$

Table 5: Calculation of $x_{1}$ by iterative method ( $q$-analogue of Numerical Methods) for different values of $q$

\begin{tabular}{|l|l|l|l|l|l|}
\hline $\mathbf{q}$ & $\mathbf{f}\left(\mathbf{q} \mathbf{x}_{\mathbf{~}}\right)$ & $\mathbf{x}_{1}$ & $\mathbf{f}\left(\mathbf{x}_{1}\right)$ & $\mathbf{f}\left(\mathbf{q}_{1}\right)$ & $\mathbf{x}_{2}$ \\
\hline $\mathbf{0 . 9 9 9 9 9 9 9}$ & $\mathbf{0 . 0 7 2 0 0 0 0 5 4}$ & $\mathbf{0 . 9 0 5 8 8 2 4 1 6}$ & $\mathbf{0 . 0 1 6 8 8 2 5 3 5}$ & $\mathbf{0 . 0 1 6 8 8 2 5 6 6}$ & $\mathbf{0 . 9 5 4 1 3 2 5 4 7}$ \\
\hline $\mathbf{0 . 9 9 9 9 9 9 9}$ & $\mathbf{0 . 0 7 2 0 0 0 5 4 4}$ & $\mathbf{0 . 9 0 5 8 8 2 9 7 9}$ & $\mathbf{0 . 0 1 6 8 8 2 3 3 8}$ & $\mathbf{0 . 0 1 6 8 8 2 6 5 5}$ & $\mathbf{0 . 9 5 4 1 3 2 6 2 1}$ \\
\hline $\mathbf{0 . 9 9 9}$ & $\mathbf{0 . 0 7 2 5 4 4 8 9 5}$ & $\mathbf{0 . 9 0 6 5 0 8 3 4 5}$ & $\mathbf{0 . 0 1 6 6 6 4 1 9 8}$ & $\mathbf{0 . 0 1 6 9 8 0 8 4 3}$ & $\mathbf{0 . 9 5 4 2 1 5 4 1 2}$ \\
\hline $\mathbf{0 . 9 9}$ & $\mathbf{0 . 0 7 7 5 2 9 0 8 8}$ & $\mathbf{0 . 9 1 2 1 7 6 3 1 3}$ & $\mathbf{0 . 0 1 4 7 4 8 6 1 6}$ & $\mathbf{0 . 0 1 7 8 8 5 7 0 7}$ & $\mathbf{0 . 9 5 5 0 6 1 0 7 4}$ \\
\hline $\mathbf{0 . 9 8}$ & $\mathbf{0 . 0 8 3 2 3 4 3 0 4}$ & $\mathbf{0 . 9 1 8 5 4 3 0 6 8}$ & $\mathbf{0 . 0 1 2 7 2 9 9 7 8}$ & $\mathbf{0 . 0 1 8 9 3 6 3 3 4}$ & $\mathbf{0 . 9 5 6 2 2 3 9}$ \\
\hline $\mathbf{0 . 9 7}$ & $\mathbf{0 . 0 8 9 1 1 2 5 7 6}$ & $\mathbf{0 . 9 2 4 9 7 8 3 6 8}$ & $\mathbf{0 . 0 1 0 8 3 4 2 5}$ & $\mathbf{0 . 0 2 0 0 3 8 2 9 6}$ & $\mathbf{0 . 9 5 7 6 4 2 6 3 8}$ \\
\hline 1.000001 & $\mathbf{0 . 0 7 1 9 9 9 4 5 6}$ & $\mathbf{0 . 9 0 5 8 8 1 7 2 7}$ & $\mathbf{0 . 0 1 6 8 8 2 7 7 5}$ & $\mathbf{0 . 0 1 6 8 8 2 4 5 8}$ & $\mathbf{0 . 9 5 4 1 3 2 4 5 7}$ \\
\hline $\mathbf{0 . 9 9 9 9 9 9 9}$ & $\mathbf{0 . 0 7 2 0 0 0 0 5 4}$ & $\mathbf{0 . 9 0 5 8 8 2 4 1 6}$ & $\mathbf{0 . 0 1 6 8 8 2 5 3 5}$ & $\mathbf{0 . 0 1 6 8 8 2 5 6 6}$ & $\mathbf{0 . 9 5 4 1 3 2 5 4 7}$ \\
\hline
\end{tabular}

Since Exact root is 1

$\left|\epsilon_{0}\right|=\left|\xi-x_{0}\right|=0.8$

$\left|\epsilon_{1}\right|=\left|\xi-x_{1}\right|=0.94118$

$\left|\epsilon_{2}\right|=\left|\xi-x_{2}\right|=0.045868$

$\left|\epsilon_{3}\right|=\left|\xi-x_{3}\right|=0.22662$

If we consider multiplicity which is 2 here then we will use Generalized Newton Raphson Method.

Table 6: Calculation of $x_{1}$ and $x_{2}$ by iterative method (q-analogue of Newton Raphson Method with multiplicity) for different values of $\mathrm{q}$

\begin{tabular}{|l|l|l|}
\hline $\mathrm{q}$ & \multicolumn{1}{|c|}{$\mathrm{x}_{1}$} & \multicolumn{1}{c|}{$\mathrm{x}_{2}$} \\
\hline 0.9999999 & 1.011765 & 1.000034249 \\
\hline 0.999 & 1.013017 & 1.000033798 \\
\hline 0.99 & 1.024353 & 0.9935928 \\
\hline 0.98 & 1.037086 & 0.985729222 \\
\hline 0.97 & 1.049957 & 0.977176327 \\
\hline 1.000001 & 1.011763 & 1.000034801 \\
\hline
\end{tabular}

$\left|\epsilon_{0}\right|=\left|\xi-\mathrm{x}_{0}\right|=0.2$

$\left|\epsilon_{1}\right|=\left|\xi-\mathrm{x}_{1}\right|=0.12 \mathrm{E}-01$

$\left|\epsilon_{2}\right|=\left|\xi-\mathrm{x}_{2}\right|=0.34 \mathrm{E}-04$

$\left|\epsilon_{3}\right|=\left|\xi-x_{2}\right|=0.74 \mathrm{E}-07$

\section{Conclusion}

q-analogue of iterative methods for solving algebraic and transcendental equations gives the same result as classical methods do but it converges more rapidly towards solution and errors associated with these methods are comparatively lesser if value of $q$ is chosen accordingly and this method is very appropriate for solving transcendental equations .By using single parameter we have to choose value of q very close to one but for double parameter we can get accurate result for most of the values of $\mathrm{q}_{1}$.Problems have been solved using $\mathrm{C}++$ Programming Language .

\section{References}

[1] F. H. Jackson, A q-form of Taylor's formula, Mess. Math., 38, 1909,62-64.

[2] G. Gasper and M. Rahman, Basic Hypergeometric Series, CambridgeUniversity Press, Cambridge, 1990

[3] Harold Exton,q-Hypergeometric Functions And Applications

[4] L.J. Slater, Generalized Hypergeometric Functions, Cambridge University Press, Cambridge, London and New York, 1966

[5] Thomas Ernst, A method for for q-Calculus, Journal of Nonlinear Mathematical Physics, 2003 